\title{
Olfactory conditioning of the sting extension reflex in honeybees: Memory dependence on trial number, interstimulus interval, intertrial interval, and protein synthesis
}

\author{
Martin Giurfa, 1,2,4 Eve Fabre, ${ }^{1,2,3}$ Justin Flaven-Pouchon, ${ }^{1,2,3}$ Helga Groll, 1,2,3 \\ Barbara Oberwallner, ${ }^{1,2,3}$ Vanina Vergoz, ${ }^{1,2,3}$ Edith Roussel, ${ }^{1,2}$ \\ and Jean Christophe Sandoz ${ }^{1,2}$ \\ ${ }^{1}$ Université de Toulouse, UPS, Centre de Recherches sur la Cognition Animale, F-31062 Toulouse Cedex 9, France; ${ }^{2}$ CNRS, Centre de \\ Recherches sur la Cognition Animale, F-31062 Toulouse Cedex 9, France
}

\begin{abstract}
Harnessed bees learn to associate an odorant with an electric shock so that afterward the odorant alone elicits the sting extension response (SER). We studied the dependency of retention on interstimulus interval (ISI), intertrial interval (ITI), and number of conditioning trials in the framework of olfactory SER conditioning. Forward ISIs (conditioned stimulus [CS] before unconditioned stimulus [US]) supported higher retention than a backward one (US before CS) with an optimum around $3 \mathrm{sec}$. Spaced trials (ITI $10 \mathrm{~min}$ ) supported higher retention than massed trials (ITI $1 \mathrm{~min}$ ) and led to the formation of a late long-term memory (I-LTM) that depended on protein synthesis. Our results reaffirm olfactory SER conditioning as a reliable tool for the study of learning and memory.
\end{abstract}

The honeybee Apis mellifera is a main invertebrate model for the study of learning and memory as it allows combining controlled conditioning protocols with a simultaneous access to the nervous system in the laboratory (Menzel 1999; Giurfa 2007). The main protocol used to this end relies on the proboscis extension reflex (PER), the appetitive reflex exhibited by a harnessed honeybee to a sugar reward (the unconditioned stimulus, or US) delivered to its antennae and mouthparts. After appropriate pairing of an odorant (the conditioned stimulus, or CS) with sucrose presentations, the bee learns to associate odorant and sugar reward so that the odorant alone elicits PER (Takeda 1961; Bitterman et al. 1983). Despite the important progress made in understanding the behavioral, cellular, and molecular bases of this appetitive learning (Menzel 1999; Giurfa 2007), until recently it has been impossible to study aversive learning in bees in such a way that behavioral records would be accompanied by access to the nervous system. This gap has been filled by a novel conditioning protocol in which individually harnessed bees learn to associate an initially neutral odorant (CS) with a mild electric shock (US) (Vergoz et al. 2007). Bees fixed individually on a metallic holder (Fig. 1A) reflexively extend their sting (sting extension response, or SER) upon delivery of an electric shock to the thorax (Núñez et al. 1997; Balderrama et al. 2002), thus showing a typical defensive response to potentially noxious stimuli (Breed et al. 2004). After successful conditioning, the odorant elicits SER, a conditioned response that can be retrieved $1 \mathrm{~h}$ after conditioning (Vergoz et al. 2007). This form of conditioning is indeed aversive as shown by the fact that bees trained in this way and transferred to the operant context of a Y-maze, where they can freely walk and choose between the shock-associated odor and a non-shock-associated odor, explicitly

\footnotetext{
${ }^{3}$ These authors contributed equally to this work.

${ }^{4}$ Corresponding author.

Email giurfa@cict.fr; fax 33-561-55-61-54.

Article is online at http://www.learnmem.org/cgi/doi/10.1101//m.1603009.
}

avoid the punished odor and choose the non-shock-associated odor $1 \mathrm{~h}$ after conditioning (Carcaud et al. 2009).

Classical features of Pavlovian conditioning protocols such as the dependence of retrieval on the interstimulus interval (ISI; the interval between CS and US onset) and intertrial interval (ITI; the interval between consecutive trial onset) have not been analyzed thus far in olfactory SER conditioning. Furthermore, although olfactory SER conditioning leads to an aversive memory that can be retrieved either in a Pavlovian (Vergoz et al. 2007) or in an operant framework (Carcaud et al. 2009) $1 \mathrm{~h}$ after conditioning, it is unknown whether it leads to the formation of robust LTMs, retrievable some days after conditioning. In the honeybee, one pairing of an odorant with sucrose (i.e., one conditioning trial) leads to an early long-term memory (e-LTM) that can be retrieved 24 to $48 \mathrm{~h}$ after conditioning. Three conditioning trials, on the other hand, lead to a stable late long-term memory (l-LTM) that can be retrieved $72 \mathrm{~h}$ or more after conditioning. Although the presence of short-term memories (STM) and medium-term memories (MTM) - retrievable just after conditioning or $1 \mathrm{~h}$ after it, respectively-have been shown in olfactory SER conditioning (Vergoz et al. 2007; Carcaud et al. 2009), no evidence thus far supports the presence of LTM after this form of learning. Here we performed a series of experiments aimed at characterizing the effect of variables such as ISI and ITI on learning and retention performance in olfactory SER conditioning. We asked whether this protocol leads to the formation of LTM and whether such a memory is protein-synthesis dependent.

Honeybee foragers were captured when leaving the hive. These individuals have a fully developed sting reflex (Burrell and Smith 1994) and exhibit higher shock responsiveness and aversive learning scores (Roussel et al. 2009). In all experiments, we followed the standard criterion used in olfactory appetitive PER conditioning, which consists of checking before conditioning and after retention tests that the unconditioned response (SER) to the US (the electric shock) is intact. This control ensures that bees have 

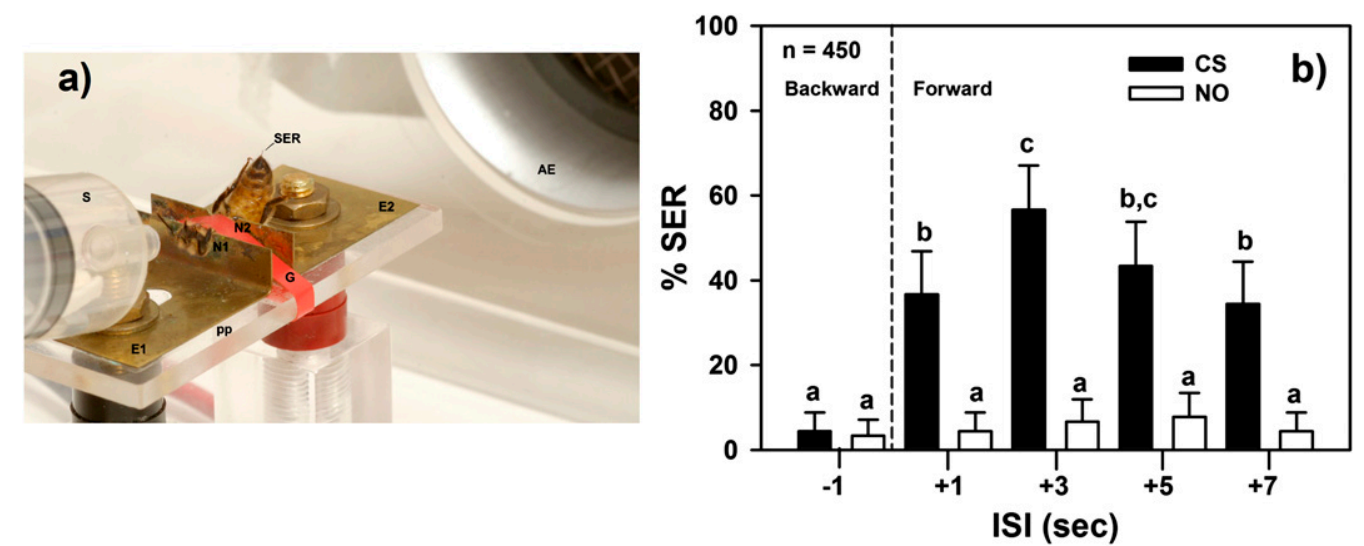

Figure 1. (A) View of a honeybee in the experimental setup. The bee is fixed between two brass plates (E1, E2) set on a Plexiglas plate (pp), with EEG cream smeared on the two notches $(\mathrm{N} 1, \mathrm{~N} 2)$ to ensure good contact between the plates and the bee, and a girdle (G) that clamps the thorax to restrain mobility. The bee closes a circuit and receives a mild electric shock $(7.5 \mathrm{~V})$ which induces the sting extension reflex (SER). An originally neutral odorant is delivered through a $20-\mathrm{ml}$ syringe $(S)$ placed $1 \mathrm{~cm}$ from the antennae. Odorant stimulation lasted $5 \mathrm{sec}$. The electric shock started 3 sec after odorant onset and lasted $2 \mathrm{sec}$ so that it ended with odorant offset. Contamination with remains of odorants used for conditioning or pheromones is avoided via an air extractor (AE) which is on continuously. (B) The effect of the ISI on retention. Percentage of SER (+95\% confidence interval) to the conditioned odor (CS; black bars) and to a novel odor (NO; white bars) as a function of ISI (interval between CS and US onset) in retention tests performed 20 min after conditioning. One ISI corresponded to a backward conditioning (US before CS; ISI -1) while the four others corresponded to forward conditioning (CS before US; ISI $+1,+3,+5$, or +7 ). Letters indicate significant differences. No retention was found at ISI -1 . Significant retention was found for forward ISIs with two ISIs, +3 and $+5 \mathrm{sec}$, yielding higher retention performance and an optimum at $+3 \mathrm{sec}$.

the potentiality to produce the conditioned response and that absence of a response during a test is due to a memory deficit and not to a motor problem. Only bees that did not show SER to the shock in these conditions were discarded. This represented $5 \%$ of the total bees conditioned and tested.

In a first experiment we studied the effect of the ISI on retention and determined the optimal ISI for SER conditioning. Using independent groups of bees, we studied the effect of five different ISIs on retention. One ISI corresponded to a backward conditioning situation as shock onset occurred $1 \mathrm{sec}$ before odor onset (ISI-1); the four other ISIs corresponded to forward conditioning situations as the odor always preceded the US, which started $1,3,5$, or $7 \mathrm{sec}$ after odor onset (ISI $+1,+3,+5$, or +7 , respectively). Each ISI group was conditioned along 10 conditioning trials spaced by $5 \mathrm{~min}$. Odor and shock delivery lasted 5 and $2 \mathrm{sec}$, respectively. Within each ISI group, two subgroups were used ( $n=45$ for each subgroup): one that experienced 1-nonanol as the CS and one that experienced 1-hexanol as the CS. Twenty minutes after the last conditioning trial, retention was determined in extinction conditions (no shock delivered). Bees were then presented with two odorants in a random succession: the CS and a novel odorant used to determine the specificity of the memory retrieved. Bees trained with 1-nonanol were presented with 1-hexanol as the novel odorant, while bees trained with 1-hexanol were presented with 1-nonanol as the novel odorant.

A total of 450 bees were used in this experiment (five ISI groups; two subgroups per ISI, one trained with 1-nonanol and the other with 1-hexanol; 45 bees per subgroup). Performance of the subgroups was pooled given the lack of significance for the factor "CS odor" $\left(F_{(1,440)}=1.50\right.$; NS) and for the interaction between the factors "CS odor" and "ISI group" $\left(F_{(4,440)}=0.09\right.$; NS). Retention performance levels varied significantly depending on ISI $\left(F_{(4,445)}=\right.$ 16.56; $P<0.0001$; Fig. 1B). They exhibited the typical bell-shaped form of classical conditioning with asymmetry between forward and backward ISIs. Indeed, no retention was found at ISI - 1 (backward conditioning), while significantly higher responses were recorded for all other ISIs (forward conditioning; NewmanKeuls post-hoc contrasts: $P<0.001$ in all cases). Two ISIs, 3 and $5 \mathrm{sec}$, yielded higher retention performance, with an optimum at
3 sec. In all cases, unspecific responses to the novel odorant were similarly low for all ISI groups $\left(F_{(1,44)}=0.59\right.$; NS), and, in the case of forward ISIs, they were significantly lower than responses to the CS $\left(F_{(1,359)}=210.34 ; P<0.00001\right.$; Fig. 2$)$. Thus, retention performance recorded 20 min after conditioning was specific for the CS and was clearly affected by ISI.

In a second experiment, we studied memory retention after olfactory SER conditioning. We asked whether SER conditioning leads to the formation of memories retrievable $1,24,48$, and $72 \mathrm{~h}$ after conditioning, the latter corresponding to l-LTM as characterized in olfactory PER conditioning (Menzel 1999). An independent group of bees was used for each retention time. Two odorants,

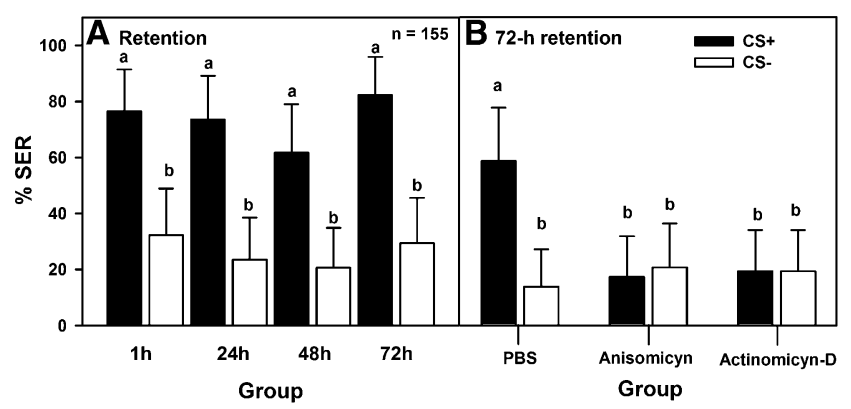

Figure 2. (A) Memory retention after SER differential conditioning (one odorant associated with shock, or CS+, vs. one odorant nonassociated with shock, or CS - ). Percentage of SER (+95\% confidence interval) to the $\mathrm{CS}+$ (black bars) and to the CS - (white bars). Four groups of bees were trained in parallel (acquisition) and tested afterward after different retention intervals ( 1 h, 24 h, 48 h, and $72 \mathrm{~h}$ post-conditioning). Each group was tested once. Letters indicate significant differences. All groups remembered the discrimination learned during training. (B) Dependency of I-LTM (72-h retention) on translation and transcription. Three groups of bees were trained in parallel (acquisition) and tested $72 \mathrm{~h}$ after the last acquisition trial and after injection of PBS, anisomycin, or actinomycin D. Each group was tested once. Letters indicate significant differences. Only the group injected with PBS (control) remembered the discrimination learned during training; inhibition of transcription (actinomycin D) or translation (anisomycin) resulted in the absence of I-LTM. 
1-hexanol and 1-nonanol, were used to condition the bees in a differential conditioning protocol (one odor associated with shock, or CS+, vs. another odor not associated with shock, or CS-) involving six CS+ and six CS- trials presented in a pseudorandom sequence. The ISI was $3 \mathrm{sec}$ (see above), and conditioning trials were spaced by $10 \mathrm{~min}$. In order to balance olfactory experiences, two subgroups were conditioned within each retention group: one for which 1-hexanol was the CS+, and 1-nonanol the CS-, and one for which the contingencies were inversed. In no case did we find significant differences between the subgroup trained with 1-hexanol as CS+ and the one that trained with 1-nonanol, so the results of both subgroups were pooled (1-h group: $F_{(1,180)}=0.94$, NS; 24-h group: $F_{(1,300)}=0.55, \mathrm{NS} ; 48$-h group: $F_{(1,345)}=0.37, \mathrm{NS}$; 72-h group: $F_{(1,345)}=1.77$, NS).

All four groups learned to discriminate the CS+ from the CS(1-h group: $F_{(1,185)}=46.30, P<0.00001 ; 24-\mathrm{h}$ group: $F_{(1,305)}=$ 44.44, $P<0.00001$; 48h-group: $F_{(1,350)}=57.44, P<0.00001 ; 72-\mathrm{h}$ group: $\left.F_{(1,350)}=52.88, P<0.00001\right)$ and reached comparable levels of discrimination at the end of training. Indeed, a within-group comparison of responses to the CS+ and the CS- in the last (sixth) conditioning trial yielded significant differences for all four groups (1-h group: $\chi^{2}=17.63, P<0.0001 ; 24$-h group: $\chi^{2}=20.90, P<$ 0.0001; 48-h group: $\chi^{2}=31.11, P<0.0001 ; 72$-h group: $\chi^{2}=25.35$, $P<0.0001)$. Neither differences in CS+ responses $\left(F_{(3,238)}=1.06\right.$; NS) nor differences in CS - responses $\left(F_{(3,238)}=1.59\right.$; NS) were found between groups at the end of training.

After conditioning, groups were tested at different retention intervals (Fig. 2A). To ensure survival at longer intervals $(24 \mathrm{~h}$, $48 \mathrm{~h}$, and $72 \mathrm{~h}$ ), bees were released from the holders and separately caged according to their retention interval. They received $50 \%$ sucrose solution ad libitum and were kept in an incubator in the dark. Three hours before retention tests they were frozen and fixed again in the holders. All groups exhibited significant retention and responded more to the $\mathrm{CS}+$ than to the $\mathrm{CS}-$. Within-group analysis by means of the McNemar test showed significant retention performance in all cases (1-h group: $\chi^{2}=20.48, P<0.0001$; 24-h group: $\chi^{2}=8.20, P<0.005 ; 48$-h group: $\chi^{2}=6.32, P<0.02 ; 72-\mathrm{h}$ group: $\left.\chi^{2}=7.61, P<0.01\right)$. Neither differences in $\mathrm{CS}+$ responses $\left(F_{(3,151)}=1.75\right.$; NS) nor differences in CS - responses $\left(F_{(3,151)}=\right.$ 0.60 ; NS) were found between groups in retention tests. These results show, therefore, that SER conditioning leads to robust LTMs that are retrievable even $3 \mathrm{~d}$ after training.

In a third experiment we asked whether the 3-d long-term memory depends on de novo protein synthesis. The conditioning procedure was identical to that of the previous experiment. In the $2 \mathrm{~h}$ following conditioning, bees were injected in the ocellar tract either with PBS (control group), anisomycin $10^{-2} \mathrm{M}$ (a translation inhibitor), or actinomycin D $1.5 \times 10^{-3} \mathrm{M}$ (a transcription inhibitor). A Harvard GC 100-10 microelectrode filled with the drug to be injected was connected to an IM 300 Narishige microinjector and used to deliver $10 \times 20 \mathrm{nl}$ into the brain. Drugs, concentrations, and injection time were chosen according to Wüstenberg et al. (1998), who showed the effectiveness of this procedure in the study of appetitive olfactory LTM and its dependency on protein synthesis. Each of these groups included two subgroups, one in which 1-hexanol was the CS+ and 1-nonanol the CS-, and one in which the contingencies were inversed (CS+: 1-nonanol; CS-: 1-hexanol). After injection, bees were marked to identify them according to the treatment received and were kept in small cages until retention tests were performed $72 \mathrm{~h}$ after the last acquisition trial.

Before injection, all three groups learned to discriminate the CS+ from the CS - (PBS group: $F_{(1,680)}=7.11, P<0.01$; anisomycin group: $F_{(1,600)}=9.99, P<0.01$; actinomycin D group: $F_{(1,350)}=6.81$, $P<0.05)$ and reached comparable levels of discrimination at the end of training. Indeed, a within-group comparison of responses to the CS+ and the CS - in the last (sixth) conditioning trial yielded significant differences for all three groups (PBS group: $\chi^{2}=$ 10.81, $P<0.01$; anisomycin group: $\chi^{2}=9.82, P<0.01$; actinomycin D group: $\left.\chi^{2}=13.14, P<0.001\right)$. Neither differences in CS+ responses $\left(F_{(2,169)}=0.18\right.$; NS) nor differences in CS - responses $\left(F_{(2,169)}=0.81\right.$; NS) were found between groups at the end of training. Thus, all three groups exhibited similar acquisition performance before drug injection.

After injection, and $72 \mathrm{~h}$ after conditioning, retention performance varied depending on treatment (Fig. 2B). Responses to CS+ differed significantly between groups $\left(F_{(2,86)}=8.54 ; P<0.001\right)$ while responses to CS - remained low and did not differ $\left(F_{(2,86)}=\right.$ 0.26 ; NS). Within-group analysis showed that retention was significant in control bees injected with PBS $\left(\chi^{2}=6.86, P<0.01\right)$ but not in bees injected either with anisomycin $\left(\chi^{2}=0\right.$; NS) or with actinomycin D $\left(\chi^{2}=0.08\right.$; NS). Thus, both translation and transcription are essential events for LTM formation and retrieval of the odor-shock association $72 \mathrm{~h}$ after training.

Finally, in a fourth experiment we studied the effect of trial number (one vs. five trials) and ITI on long-term retention. Two single-trial groups were studied: one in which the CS preceded the US by $3 \mathrm{sec}$ (one-trial forward, or 1CT F), and one in which the US preceded the CS by $1 \mathrm{sec}$ (one-trial backward, or 1CT B). In addition, two forward five-trial groups were studied: one in which the ITI was $1 \mathrm{~min}$ (massed trials; 5CT F 1min), and one in which the ITI was $10 \mathrm{~min}$ (spaced trials; 5CT F 10min). Each ITI group experienced 1-nonanol as the CS. In retention tests, bees were presented with the CS and with 1-hexanol as a novel odor used to test the specificity of the memories retrieved. Retention tests were performed $24 \mathrm{~h}$ and $72 \mathrm{~h}$ after conditioning. An independent group of bees was used in each case. Thus, our design involved four ITI groups (1CT B; 1CT F; 5CT F 1min, and 5CT F 10min) and two subgroups per ITI (24-h test and 72-h test). In total eight groups, amounting to 330 bees, were used ( 40 bees per group on average).

Figure 3 shows retention performance $24 \mathrm{~h}$ (Fig. 3A) and $72 \mathrm{~h}$ after conditioning (Fig. 3B). At both times, retention varied significantly between groups. Responses to the conditioned odor (CS) varied between ITI groups both at $24 \mathrm{~h}\left(F_{(3,162)}=14.37 ; P<\right.$ $0.0001)$ and $72 \mathrm{~h}\left(F_{(3,162)}=16.54 ; P<0.0001\right)$ while responses to the novel odor $(\mathrm{NO})$ remained low and nonsignificant both at $24 \mathrm{~h}\left(F_{(3,162)}=0.88 ; \mathrm{NS}\right)$ and $72 \mathrm{~h}\left(F_{(3,162)}=2.42 ; \mathrm{NS}\right)$. Twenty-four hours after training, no significant retention was found in the group conditioned with five massed trials, although performance was marginally nonsignificant $\left(\chi^{2}=3.37, P=0.07\right)$. In contrast, the group conditioned with five spaced trials exhibited significant retention $\left(\chi^{2}=13.79 ; P<0.001\right)$, thus showing that ITI has a significant effect per se on 24-h olfactory retention. Neither conditioning with one backward trial $\left(\chi^{2}=0\right.$; NS) nor conditioning with one forward trial $\left(\chi^{2}=0.90\right.$; NS) supported significant retention.

Seventy-two hours after training, significant retention was again found in the group conditioned with five spaced trials $\left(\chi^{2}=\right.$ $10.62, P<0.01)$. The group conditioned with five massed trials exhibited now significant retention $\left(\chi^{2}=4.05, P<0.5\right)$, higher than that of both one-trial conditioning groups, but lower than that of the five-trial spaced group (Group 1CT B: $\chi^{2}=0.25$, NS; Group 1CT F: $\chi^{2}=0.17$, NS). Thus, comparing the group conditioned with one forward trial and the groups conditioned with five forward trials, massed and spaced, clearly shows an effect of trial number at $72 \mathrm{~h}$. As for the 24-h test, significant differences between both groups conditioned with five forward trials showed that ITI significantly affected olfactory retention.

Our work shows, therefore, that olfactory SER conditioning has the typical features of Pavlovian conditioning, namely a dependence of retention on ISI, on ITI, and on the number of conditioning trials. Forward ISIs supported higher retention levels 


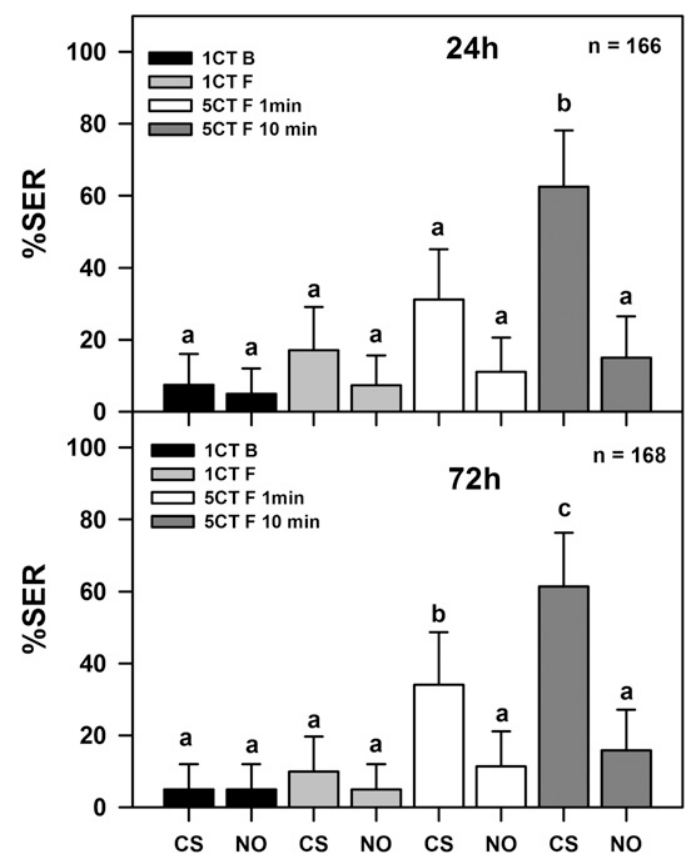

Figure 3. Trial number, ITI, and long-term retention. Percentage of SER (+95\% confidence interval) to the conditioned odor (CS) and to a novel odor (NO) as a function of the number of trials (1CT vs. 5CT) and ITI (interval between successive trial onset; 1 vs. $10 \mathrm{~min}$ ) in retention tests performed $24 \mathrm{~h}$ or $72 \mathrm{~h}$ after conditioning. One-trial groups were either forward (the CS preceded the US) or backward (the US preceded the CS). Each group was tested once. Letters indicate significant differences. Upper graph: performance in retention tests performed $24 \mathrm{~h}$ after conditioning; lower graph: performance in retention tests performed $72 \mathrm{~h}$ after conditioning.

than a backward one, and an optimal ISI was found around 3 sec. One- and multiple-trial backward conditioning induces conditioned inhibition in appetitive olfactory conditioning of PER, which can be demonstrated if acquisition of a conditioned response during subsequent pairings of the putative inhibitory CS with the US is retarded (retardation of acquisition) (Hellstern et al. 1998). However, such an effect was mainly visible for an ISI of -15 sec, while no inhibitory effect was found for shorter intervals, such as $-6 \mathrm{sec}$ (Hellstern et al. 1998). In our case an ISI of $-1 \mathrm{sec}$ was used, so that, if conditioned inhibition also occurs in the framework of olfactory SER conditioning, and if it follows similar principles as in olfactory PER conditioning, it should be negligible. In olfactory PER conditioning, optimal retention after a single conditioning trial is reached when the CS precedes the US (forward conditioning) by 5 to $1 \mathrm{sec}$ (Menzel and Bitterman 1983). In mechanosensory PER conditioning, the retention curve found after one conditioning trial shows the same typical bell-shaped form with a maximum of $\sim 3$ - to 4-sec ISI (Giurfa and Malun 2004). These coincident results suggest that, in order to learn different kinds of stimulus associations, bees require contiguity between events that should not exceed a few seconds.

Long-term retention was affected both by trial number and by ITI. One conditioning trial was unable to produce either e-LTM $(24 \mathrm{~h})$ or l-LTM $(72 \mathrm{~h})$. In contrast, five conditioning trials, massed or spaced, yielded l-LTM (at $72 \mathrm{~h}$ ), thus suggesting that aversive events need to be confirmed in order to be stored as memories. Spaced trials (ITI $10 \mathrm{~min}$ ) resulted in better retention performance both $24 \mathrm{~h}$ and $72 \mathrm{~h}$ after conditioning, while massed trials (ITI $1 \mathrm{~min}$ ) yielded intermediate-level retention at $72 \mathrm{~h}$ but not at $24 \mathrm{~h}$. This result is intriguing as, in analogy with olfactory PER conditioning, one would have expected to observe retention at $24 \mathrm{~h}$ but not at $72 \mathrm{~h}$ after massed conditioning. However, extensive discussion on this difference should be precluded as retention values at 24 and $72 \mathrm{~h}$ are just below and above statistical significance. Although this result remains unexplained, we verified here the classical distinction between massed and spaced trials in terms of their efficiency to support LTM. Massed trials imply rapid successions of affirmative information that could interfere with each other in terms of the storage processes that each one triggers. In appetitive PER conditioning, a higher sensitivity of the memory trace to conflicting or affirmative information can be found $\sim 2-3$ min after conditioning (Menzel et al. 1974; Erber et al. 1980). We have not tested the effect of an ITI of 2-3 min on retention after SER conditioning, but we suggest that a similar detrimental effect was found here for an ITI of $1 \mathrm{~min}$.

As experiments were not performed simultaneously, two different conditioning protocols were used in our work: absolute (first and fourth experiments: CS+) and differential (second and third experiments: CS+ vs. CS-) conditioning. This fact does not invalidate the findings reported, as we analyzed each experiment per se and did not compare retention levels between experiments. It would be interesting nevertheless to compare acquisition, discrimination, and retention resulting from these two protocols as is done in the visual modality (Giurfa et al. 1999; Giurfa 2004). We predict that differential conditioning will lead to better acquisition and discrimination performance, as in the case of achromatic patterns and color stimuli (Giurfa et al. 1999; Giurfa 2004).

Olfactory SER conditioning can induce a robust and stable l-LTM that relies on protein synthesis as it depends on both translation and transcription. Our results show that bees have the capacity to remember aversive experiences long after those experiences took place. There are multiple biological contexts in which such capacity could be applied; for example, foragers could avoid returning to food places in which negative experiences, or eventually unfulfilled expectations, occurred, thus enhancing foraging efficiency. Furthermore, aversive memories could help in organizing defensive responses against enemies whose odors have been previously experienced. It may thus be adaptive to memorize and remember for long periods the smell of predators in order to exhibit appropriate defensive responses.

\section{Acknowledgments}

We thank two anonymous reviewers for useful comments on a previous version of the manuscript. This work was supported by the French National Research Agency (Project ANR-BLAN083_337040; INSAVEL), the French Research Council (CNRS), and Paul-Sabatier University. H.G. was supported by the Austrian Bundesministerium für Bildung, Wissenschaft, und Kultur; the Erasmus Program; and the University of Wien (KWA fellowship). B.O. was supported by the Erasmus Program.

\section{References}

Balderrama N, Núñez JA, Guerrieri F, Giurfa M. 2002. Different functions of two alarm substances in the honeybee. J Comp Physiol [A] 188: 485-491.

Bitterman ME, Menzel R, Fietz A, Schäfer S. 1983. Classical conditioning of proboscis extension in honeybees (Apis mellifera). J Comp Psychol 97: 107-119.

Breed MD, Guzmán-Novoa E, Hunt GJ. 2004. Defensive behavior of honey bees: Organization, genetics, and comparisons with other bees. Annu Rev Entomol 49: 271-298.

Burrell BD, Smith BH. 1994. Age-related but not caste-related regulation of abdominal mechanisms underlying the sting reflex of the honey bee, Apis mellifera. J Comp Physiol [A] 174: 581-592.

Carcaud J, Roussel E, Giurfa M, Sandoz JC. 2009. Odour aversion after olfactory conditioning of the sting extension reflex in honeybees. J Exp Biol 212: 620-626.

Erber J, Masuhr T, Menzel R. 1980. Localization of short-term memory in the brain of the bee, Apis mellifera. Physiol Entomol 5: 343-358. 
Giurfa M. 2004. Conditioning procedure and color discrimination in the honeybee Apis mellifera. Naturwissenschaften 91: 228-231.

Giurfa M. 2007. Behavioral and neural analysis of associative learning in the honeybee: A taste from the magic well. J Comp Physiol [A] 193: $801-824$

Giurfa M, Malun D. 2004. Associative mechanosensory conditioning of the proboscis extension reflex in honeybees. Learn Mem 11: 294302.

Giurfa M, Hammer M, Stach S, Stollhoff N, Müller-Deisig N, Mizyrycki C. 1999. Pattern learning by honeybees: Conditioning procedure and recognition strategy. Anim Behav 57: 315-324.

Hellstern F, Malaka R, Hammer M. 1998. Backward inhibitory learning in honeybees: A behavioral analysis of reinforcement processing. Learn Mem 4: 429-444.

Menzel R. 1999. Memory dynamics in the honeybee. J Comp Physiol A Neuroethol Sens Neural Behav Physiol 185: 323-340.

Menzel R, Bitterman ME. 1983. Learning of honeybees in an unnatural situation. In Behavioral physiology and neuroethology: Roots and growing points (ed. F Huber, H Markl), pp. 206-215. Springer, Berlin, Germany.
Menzel R, Erber J, Masuhr T. 1974. Learning and memory in the honeybee. In Experimental analysis of insect behaviour (ed. L Barton-Browne), pp. 195-217. Springer, Berlin, Germany.

Núñez JA, Almeida L, Balderrama N, Giurfa M. 1997. Alarm pheromone induces stress analgesia via an opioid system in the honeybee. Physiol Behav 63: 75-80.

Roussel E, Carcaud J, Sandoz JC, Giurfa M. 2009. Reappraising social insect behavior through aversive responsiveness and learning. PLoS One 4: e4197. doi: 10.1371/journal.pone.0004197.

Takeda K. 1961. Classical conditioned response in the honey bee. J Insect Physiol 6: 168-179.

Vergoz V, Roussel E, Sandoz JC, Giurfa M. 2007. Aversive learning in honeybees revealed by the olfactory conditioning of the sting extension reflex. PLoS One 2: e288. doi: 10.1371/journal.pone. 0000288.

Wüstenberg D, Gerber B, Menzel R. 1998. Long- but not medium-term retention of olfactory memories in honeybees is impaired by actinomycin D and anisomycin. Eur J Neurosci 10: 2742-2745.

Received August 22, 2009; accepted in revised form October 5, 2009. 


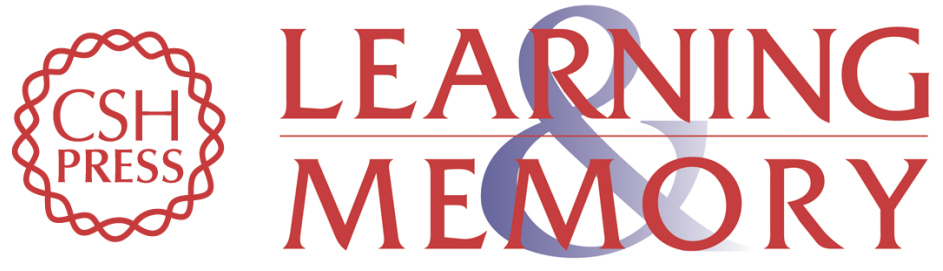

\section{Olfactory conditioning of the sting extension reflex in honeybees: Memory dependence on trial number, interstimulus interval, intertrial interval, and protein synthesis}

Martin Giurfa, Eve Fabre, Justin Flaven-Pouchon, et al.

Learn. Mem. 2009, 16:

Access the most recent version at doi:10.1101/lm.1603009

References This article cites 17 articles, 3 of which can be accessed free at: http://learnmem.cshlp.org/content/16/12/761.full.html\#ref-list-1

License

Email Alerting

Receive free email alerts when new articles cite this article - sign up in the box at the Service top right corner of the article or click here. 